\title{
Dieulafoy's disease associated with early gastric cancer
}

\author{
O Leone, M Zanelli, D Santini, F Minni, D Marrano
}

\begin{abstract}
In the past different terms have been used to define the vascular malformations of Dieulafoy's disease - for example, calibre persistent artery of the stomach, cirsoid aneurysm and gastric atherosclerosis. A case of Dieulafoy's disease is described in a 41 year old man, who presented with symptoms of anaemia and melaena, with particular attention paid to the morphological characterisation of the vascular histological lesions. Intimal hyperplasia with a non-concentric proliferation of myointimal cells, areas of muscular degeneration, aspects of vascular neoformation of the arterial wall, and other findings are reported. An association between an early diffuse adenocarcinoma and parietal anomalies of Dieulafoy's disease is illustrated.

(f Clin Pathol 1995;48:267-270)
\end{abstract}

Keywords: Dieulafoy's disease, adenocarcinoma.

Dieulafoy's disease is an arterial malformation of the upper stomach which usually occurs in middle-aged and elderly men without any relevant family history or any previous gastrointestinal symptomatology. Its typical clinical presentation is life threatening, massive and recurrent gastric bleeding.

In the medical literature there is a lot of confusion about the terminology of vascular malformations and many terms-for example, angiodysplasia, vascular ectasia, arteriovenous malformation, haemangioma, and angioma, have been used to describe this condition. In 1976 Moore et $a l^{2}$ tried to divide gastrointestinal arteriovenous malformations into three types, a classification further modified by Lewi et $a l^{3}$ and Fowler et al. ${ }^{4}$ However, these classifications of gastrointestinal vascular malformations are not sufficient for a clear definition of the pathology. Different terms have also been used to describe Dieulafoy's vascular malformation - for example, calibre persistent artery of the stomach, cirsoid aneurysm, submucosal arterial malformation, gastric arteriosclerosis, and peptic ulcer of peculiar location; however, Dieulafoy's vascular malformation is the currently accepted term. This condition was first described by Gallard ${ }^{5}$ in
1884 as submucosal miliary aneurysms; then in 1889 Dieulafoy $^{6}$ designated it exulceratio simplex believing that this lesion was an early stage of peptic ulceration. Recently, it has been interpreted as a congenital anomaly characterised by a single large, tortuous, and calibre persistent submucosal artery with no evidence of vasculitis, atherosclerosis, or aneurysm formation. ${ }^{78}$ The cause of bleeding is believed to be a mucosal erosion resulting from an artery protruding into the overlying mucosa or from focal gastritis, leading to exposure of the vessel. The mucosal ulcer, localised along the smaller curvature within $6 \mathrm{~cm}$ of the gastro-oesophageal junction, ${ }^{9}$ is usually small and shallow.

Here, we present a report of Dieulafoy's disease focusing particularly on the histopathological aspects.

\section{Methods}

A 41 year old man was admitted to St Orsola Hospital, Bologna with symptoms of anaemia (asthenia, dizziness) and melaena. There was no relevant family history, or excessive alcohol or aspirin intake. For about seven to eight months the patient had been suffering from an epigastric pain, beginning a few hours after meals and lasting through the night, but which disappeared on ingestion of food. At admission, he was found to have a haemoglobin concentration of $9 \cdot 1 \mathrm{~g} / \mathrm{dl}$, a red blood cell count of $3.01 \times 10^{6} / \mathrm{ml}$, a haematocrit of $26 \%$, a mean corpuscular volume of $86 \mathrm{~m}^{3}$, a white blood cell count of $9.76 \times 10^{3} / \mathrm{ml}$, and a platelet count of $169 \times 10^{3} \mathrm{ml}$.

An urgent gastroscopy revealed a subcardial gastric ulcer (diameter $1.5 \mathrm{~cm}$ ) with irregular edges and profuse bleeding. After transfusional therapy and antiulcer treatment (ranitidine), a repeat gastroscopy revealed that the ulcer had healed and the presence of chronic gastritis with no evidence of active bleeding. The patient was discharged and maintained on antiulcer treatment (omeprazole). After further bleeding with a melaena, the patient was readmitted to the surgical department and a gastroscopy showed a gastric ulcer in a subcardial location on the posterior medial wall, with evidence of recent bleeding. Analysis of biopsy specimens revealed atrophic chronic gastritis with diffuse intestinal metaplasia. The patient's ulcer appeared to be healing well on gastroscopy but 

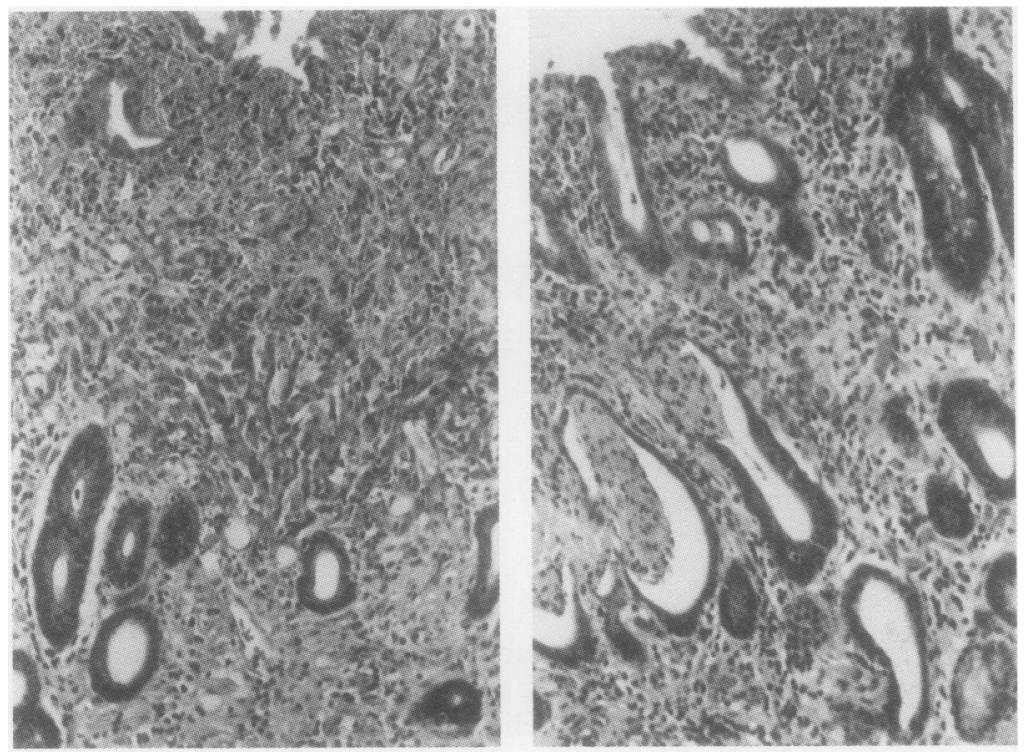

$A$

$B$

Figure 1 A: Gastric mucosa with a mild ischaemia; B: scattered signet ring cells descending from the lower part of the glandular necks.

the examination of the biopsy specimens disclosed the presence of diffuse carcinoma (microglandular type). The patient subsequently underwent laparotomy, gastrectomy and splenectomy.

The excised stomach measured $13 \mathrm{~cm}$ along the smaller and $29 \mathrm{~cm}$ along the larger curvature with an annexed duodenal tract of $2 \mathrm{~cm}$. On gross examination, the gastric mucosa was widely haemorrhagic, especially in the fundus and body regions and a retracting star-shaped area measuring $1.4 \mathrm{~cm}$ in diameter was present $4-5 \mathrm{~cm}$ from the proximal resection margin. At sectioning, this area showed focal fibrous tissue localised to the mucosa and submucosa. A second, slightly depressed area (diameter $1.5 \mathrm{~cm}$ ) was evident $0.2 \mathrm{~cm}$ from the proximal resection margin, while the antral mucosa showed diffuse bulging areas. The star-shaped and slightly depressed areas were sampled.

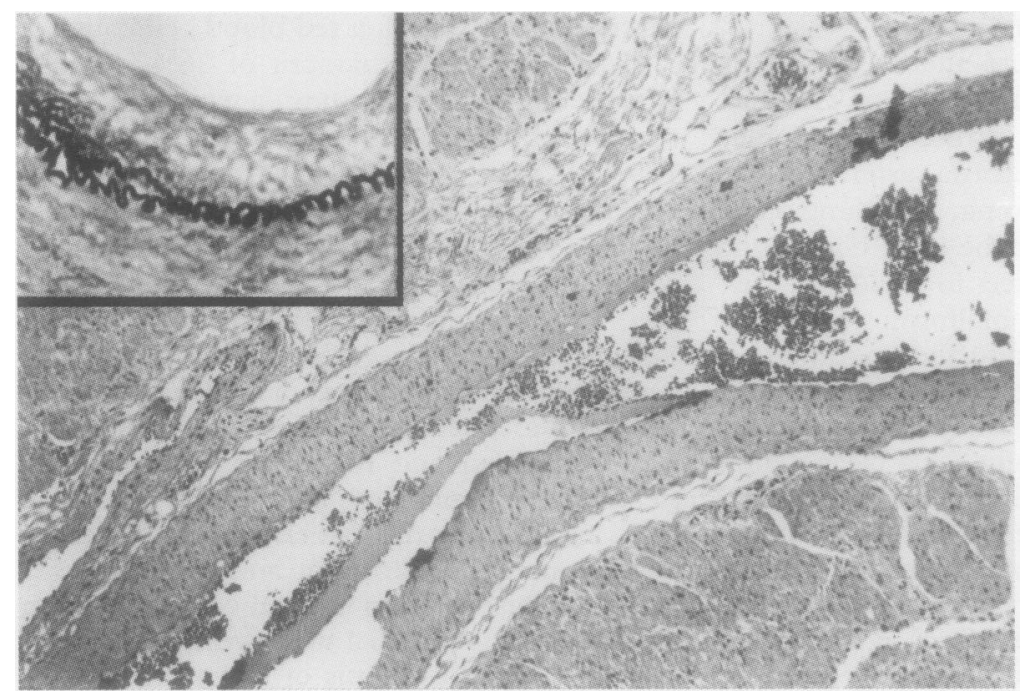

Figure 2 Photomicrograph showing the large artery running through the gastric muscle coat (haematoxylin and eosin $\times 100$ ). The insert shows focal reduplication of arterial lamina elastica (Van Gieson $\times 400)$.
Other samples were obtained from the antral region, local lymph nodes, spleen, and omentum.

All specimens were fixed in $10 \%$ buffered formalin, embedded in paraffin wax and $2 \mu \mathrm{m}$ sections were stained using haematoxylin and eosin, Van Gieson and Masson's trichrome methods. Immunohistochemical staining for keratin (clone MNF 116; Dako, Glostrup, Denmark) was performed using the Streptavidin-biotin immunoperoxidase method.

\section{Results}

Morphological examination of specimens showed the following histopathological findings. The grossly depressed areas showed reepithelialisation with residual erosions. The remaining mucosa was thickened and oedematous with diffuse intestinal metaplasia and active chronic gastritis. In the lamina propria aspects of ischaemia (fig 1A) such as subacute necrosis, fibrosis, and granulation tissue close to the muscularis mucosae were observed. In the submucosa, vessels (arteries and veins) close to focal areas of haemorrhagic effusion were enlarged and filled with serum, erythrocytes and granulocytes. The muscularis mucosae was focally thickened and dissociated by lymphoid aggregates and penetrating arterioles. The muscle coat also appeared to be disrupted by frail connective tissue and arterial vessels. Another characteristic histological feature was the presence of an unusually large artery (fig 2 ), with intimal non-concentric hyperplasia, in the muscularis and submucosa and in close contact with the mucous membrane. Arterial muscle layer fibres were mixed with fibrous tissue: in these vessels the stained elastic tissue had normal internal lamina elastica with focal areas of thickening and reduplication (fig 2 insert).

The intima of the arteriolar walls showed circumscribed thickening (fig 3B) with vacuolated cells, focal aspects of vascular neoformation (fig 3A) and areas of degeneration (muscle fibre vacuolation). Intimal hyperplasia with a non-concentric proliferation of myointimal cells was present in the arterioles penetrating through the muscularis mucosae.

Some veins had rather thick walls and dissociated elastic fibres (fig 3C). An early intramucosal, signet-ring cell, gastric cancer was noted: microfoci of scattered signet-ring cells (fig 1B) in close contact with the lower part of the glandular necks were detected in the mucosa surrounding the re-epithelialised starshaped area. We think that this aspect corresponds to the "early gastric cancer signetring cell drippings" studied by Grundmann. ${ }^{10}$

\section{Discussion}

Dieulafoy's disease, as described in various reports, is a clinical rather than a pathological entity: the unchanging clinical feature is the presence of massive, recurrent bleeding in middle-aged and elderly men with no family history or any previous gastrointestinal symptomatology. Vascular abnormalities of the 

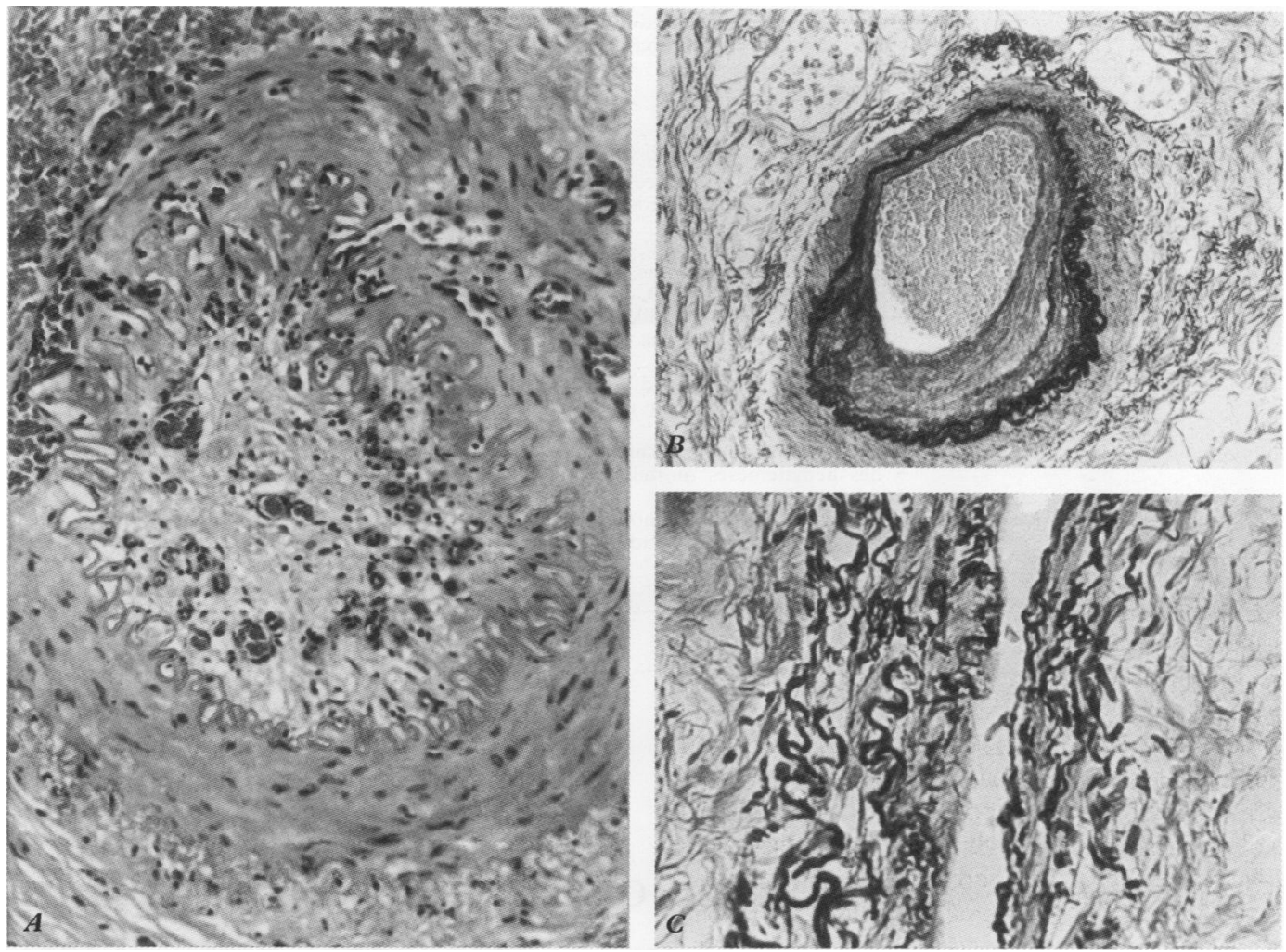

Figure 3 A: Thickened intima of an arteriole with aspects of vascular neoformation (Masson trichrome $\times 250$ ); $B$ : focal circumscribed thickening of the arteriolar wall with corresponding fragmentation of the lamina elastica ( $\times 100)$; C: segment of a thick venous wall with dissociated elastic fibres (Van Gieson $\times 400)$.

gastric wall and a small and shallow mucosal ulcer, localised to the upper stomach are the pathological features invariably observed. In the literature vascular malformations include a typical single large artery as well as thin-walled venous vessels, but a common morphological aspect does not exist. Therefore, different types of vascular anomalies have been reported and histopathological lesions range from venous to arterial alterations, as noted by Geoffrey et al. ${ }^{11}$ However, in our report, in accordance with other studies ${ }^{12}$ we noted that some pathological features occur more frequently. The architecture of the wall of the large artery is mainly normal, except for focal areas of intimal hyperplasia and duplication of the internal elastic lamina. The enlarged and tortuous submucosal vessels also show intimal non-concentric hyperplasia caused by focal myointimal cell proliferation.

Originally, Dieulafoy's disease was thought to be related to an acquired aneurysm of an atherosclerotic submucosal vessel, ${ }^{5}$ but microscopic examination does not support this view because the vessels do not show any signs of aneurysm formation, such as an arterial wall made up of fibrous tissue with no evidence of a normal arterial structure. In the same way vascular anomalies seem to be different from the telangiectasies characteristic of OslerRendu-Weber syndrome because of the absence of enlarged and thin-walled venules and capillaries. Moreover, microscopic examination did not disclose any evidence of atherosclerosis, as no typical lesions-for example, muscular cells plunging into a dense collagenous weft with several elastic fibres and/or rare foamy cells filled with fat, were observed. Vasculitis was excluded because of the lack of necrosis and lymphoid penetration of the vessel walls; the identification of active inflammatory changes in the vessel wall is considered obligatory for a histological diagnosis of arteritis. Fragmentation of the internal elastic lamina is such a constant feature of aging in all arteries that its presence alone is not suggestive of active or healed arteritis. ${ }^{13}$

In conclusion, in our opinion this study is important as we attempted to examine the histological lesions in detail. As attention has been mainly focused on the clinico-endoscopic aspects of Dieulafoy's disease in the literature, we feel we have provided further information on the morphological characteristics of this disease. We would like to underline three other points: (1) the absence of signs of atherosclerosis, aneurysm formation or vasculitis; (2) the mixture of arterial and venous anomalies as a distinctive histopathological characteristic of Dieulafoy's disease in addition to the calibre persistent submucosal arteries; and (3) gastric wall anomalies such as oedema, thickening of the submucosa, dissociation of the muscularis mucosae and muscle coat, which occur as a consequence of the vascular malformations and which could upset the trophism of the gastric wall. Finally, we would like to point out that, in the context of regenerating mucosa with aspects of ischaemic injury related to the vascular malformations, "early gastric cancer signet ring cell drippings" should be considered. 
The authors are greatly indebted to Professor A M Mancini for his helpful suggestions and criticism. Thanks also to A Busi for the photographic work.

1 Scully R, Mark EJ, McNeely WF. Case records of the Massachussets General Hospital. N Engl F Med 1991;10: 1086-96.

2 Moore JD, Thompson NW, Appelman JD, Foley D. Arteriovenous malformations of the gastrointestinal tract. Arch Sur 1976:111:381-9.

3 Lewi HJE Gledhill T, Gilmour HM, Buist TAS A teriovenous malformations of the intestine. Surg Gynecol teriovenous malformation

4 Fowler DL, Fortin D, Wood WG, Pinkerton JA Jr, Koontz PG Jr. Intestinal vascular malformations. Surgery 1979; PG Jr. Intestir

5 Gallard T. Aneurysmes miliaires de l'estomac, donnant lieu a des hematemeses mortelles. Bull Soc Med Hop Paris $1884 ; 1: 84-91$.

6 Dieulafoy G. Exulceratio simplex: l'intervention chirurgicale dans les hematemeses foudroyantes consecutives a l'ex- ulceration simplex de l'estomac. Bull Acad Med 1989;39: 49-84.

7 Mikò TL, Thomàzy VA. The caliber persistent artery of the stomach: an unifying approach to gastric aneurysm, Dieulafoy's lesion, and submucosal arterial malformation. Hum Pathol 1988;19:914-21.

8 Mortesen NJMcC, Mountford RA, Davies JD, Jeans WD. Dieulafoy's disease: a distinctive arteriovenous malformation causing massive gastric haemorrhage. $\mathrm{Br} \mathcal{F}$ Surg 1983;70:76-8.

9 Veldhuyzen van Zanten SJO, Bartelsman JFWM, Shipper MEI, Tytgat GNJ. Recurrent massive haematemesis from Dieulafoy's vascular malformations-a review of 101 cases. Gut 1986;27:213-22.

10 Grundmann E. Histologic types and possible initial stages in early gastric carcinoma. Beitrage zur Pathologie 1975; 154:256-80.

11 Mower GA, Whitehead R. Gastric hemorrhage due to ruptured arteriovenous malformation (Dieulafoy's disease). Pathology 1986;18:54-7.

12 Jaspersen D, Gaster CB, Koerner TH, Hammar CH. Doppler-controlled injection treatment of Dielafoy's disease. f Gastroenterol Hepatol 1993;8:267-9.

13 Hamrin B. Polymialgia arteritica. Acta Med Scand 1972;7:533.

\title{
Importance of hepatic artery node involvement in patients with colorectal liver metastases
}

\author{
M J Dworkin, S Earlam, C Fordy, T G Allen-Mersh
}

Department of Surgery,

Charing Cross and

Westminster Medical

School, London

M J Dworkin

$S$ Earlam

C Fordy

T G Alllen-Mersh

Correspondence to:

Mr T G Allen-Mersh,

Department of Academic

Surgery,

Chelsea and Westminster

Hospital,

369 Fulham Road,

London SW10 9NH.

Accepted for publication 25 August 1994

\begin{abstract}
Hepatic artery lymph node (HALN) involvement is an adverse prognostic factor in patients treated for colorectal liver metastases. The prevalence of HALN positivity for mid-gut and hind-gut derived colonic tumours, for differing amounts of liver involvement, and for Dukes' $A$ and B versus Dukes' C primary tumours was compared in 75 patients with colorectal liver metastases. All patients whose primary tumours did not invade lymph nodes (Dukes' A or B) had liver metastases that did not involve local hepatic nodes, regardless of the extent of the disease within the liver. This suggests that factors controlling metastasis are not identical with those which control lymphatic invasion in colorectal cancer. HALN positive patients may benefit less from treatment because they are significantly more likely to have both a greater burden of disease within the liver and a tumour with greater lymph invasive potential than patients with HALN negative liver metastases.

(F Clin Pathol 1995;48:270-272)
\end{abstract}

Keywords: Liver metastases, colorectal cancer, hepatic artery lymph node.

Hepatic artery lymph node (HALN) involvement is an adverse prognostic factor in patients being treated by resection ${ }^{1}$ or chemotherapy ${ }^{2}$ for colorectal liver metastases. How- ever, the route and mechanism of HALN involvement is uncertain.

There are two possible routes of involvement. Firstly, by direct extension of disease along the para-aortic lymph nodes from local lymph nodes draining the primary colonic tumour. If this were the case, then the prevalence of HALN positivity would be greater where the primary tumour and associated local nodes were closer to the hepatic artery-that is, primary tumours arising in mid-gut derived colon supplied by the superior mesenteric artery, than if the primary tumour and associated nodes arose more caudally in the hind-gut, which is supplied by the inferior mesenteric artery. ${ }^{3}$ Secondly, HALN involvement could occur as a result of lymphatic spread directly from the haematogenously derived liver metastases into the local lymph nodes draining the liver. ${ }^{4}$

We have examined this by measuring the prevalence of HALN positivity in patients with colorectal liver metastases and correlating this with primary tumour location in the colon and with the extent of liver metastases.

\section{Methods}

Patients with primary colorectal carcinoma and either synchronous or metachronous liver metastases undergoing hepatic artery infusional chemotherapy were prospectively studied. At the time of laparotomy for insertion of a hepatic artery catheter, hepatic artery lymph nodes adjacent to the junction between the common 